\title{
KRAS, BRAF and PIK3CA mutations in human colorectal cancer: Relationship with metastatic colorectal cancer
}

\author{
HONG-TAO LI ${ }^{*}$, YUAN-YUAN LU*, YAN-XIN AN, XIN WANG and QING-CHUAN ZHAO \\ State Key Laboratory of Cancer Biology and Xijing Hospital of Digestive Diseases, The Fourth Military \\ Medical University, \#127 Changle West Road, Xi'an, Shaanxi Province 710032, P.R. China
}

Received November 4, 2010; Accepted January 10, 2011

DOI: $10.3892 /$ or.2011.1217

\begin{abstract}
Many abnormal gene expressions and dysregulated signaling pathways have been found in human colorectal cancer. Activating mutations of the KRAS, BRAF or PIK3CA oncogenes are frequently found in colorectal cancer. The aim of the study was to investigate the molecular occurrence of KRAS, BRAF and PIK3CA mutations in the colorectal tumorigenesis and to study the association of these events with clinicopathological parameters. In our study, DNA was extracted from 200 cases of human colorectal cancer tissue samples. KRAS, BRAF and PIK3CA mutation analysis was performed by PCR and pyrosequencing. Using statistical methods, we analyzed the relationships between the gene mutations and clinicopathological parameters. KRAS point mutations were detected in $63 / 200$ patients $(31.5 \%$ ), with codon 12 mutations in 52/200 patients (26\%), codon 13 mutations in $10 / 200$ patients $(5 \%)$ and codon 12.13 bi-mutations in $1 / 200$ patients $(0.5 \%)$. The V600E mutations of BRAF were detected in $14 / 200$ patients $(7 \%)$. PIK3CA point mutations (exon 9, exon 20) were detected in 25/200 (12.5\%) patients, exon 9 mutatons in 12/200 patients $(6 \%)$ and exon 20 mutations in $13 / 200(6.5 \%)$. Our study suggested that both KRAS and BRAF mutations are exclusive, but KRAS and PIK3CA mutations are coexistent. The mutational status of BRAF did not correlate with Dukes' staging, histological type, age and gender. However, strong associations were found between KRAS, PIK3CA mutations and Dukes' staging (staging D, 12/25, 48\%). Notably, our data indicated that colorectal cancers with KRAS and PIK3CA bi-mutations are more likely to develop into liver metastasis.
\end{abstract}

Correspondence to: Dr Qingchuan Zhao or Dr Xin Wang, State Key Laboratory of Cancer Biology and Xijing Hospital of Digestive Diseases, The Fourth Military Medical University, \#127 Changle West Road, Xi'an, Shaanxi Province 710032, P.R. China

E-mail: qingchuanzhao@163.com

E-mail:wangx@fmmu.edu.cn

*Contributed equally

Key words: KRAS, BRAF, PIK3CA, mutation, metastatic colorectal cancer

\section{Introduction}

Colorectal cancer (CRC) is a multigene disease. Many abnormal gene expressions and dysregulated signaling pathways play important roles in tumorigenesis and tumor progression of CRC (1-4). EGFR is a transmembrane tyrosine kinase receptor that, on ligand binding, triggers two main signaling pathways. These include the RAS-RAF-MAPK axis, which is mainly involved in cell proliferation, and the PI3K-PTEN-AKT pathway, which is mainly involved in cell survival and motility $(2,5)$. Mutation in KRAS, BRAF or PIK3CA results in continuous activation of the downstream RAS-MAPK or PI3K pathways, regardless of whether the EGFR is activated $(6,7)$. Mutation of KRAS, BRAF or PIK3CA oncogenes is crucial to the progression of colorectal cancer (8).

Many studies have indicated that KRAS mutations are found earlier in tumor progression and occur in about 30-35\% of CRC $(9,10)$. Mutations in BRAF and PIK3CA are around $5-10 \%$ and $10-20 \%$ (11-13). Mutations in KRAS and BRAF are mutually exclusive, but KRAS and PIK3CA mutations may coexist within the same tumor (8). It is well known that molecular events resulted in the development of CRC. Little is known the relationship between these molecular events and clinical pathological parameters (age, gender, Dukes' staging, histological type and TNM), especially in metastatic colorectal cancer.

Recently, cetuximab, an IgG1 monoclonal antibody to the epidermal growth factor (EGFR), is effective in combination with chemotherapy in the first-line treatment of KRAS wild-type metastasis colorectal cancer (mCRC). However, the overall survival of $\mathrm{mCRC}$ is still relatively poor, and there is a urgent need for early and more effective therapies. Therefore, predictive and prognostic factors are required urgently and would help physicians to predict the risk of metastasis and to enact the personalized treatment for colorectal cancer patients.

In the present study, we aimed to investigate the molecular occurrence of KRAS, BRAF and PIK3CA mutations in the colorectal cancer patients and to study the association of these events with clinicopathological parameters. Notably, we also tried to explore the association of KRAS, PIK3CA bi-mutations with $\mathrm{mCRC}$. We expected to find some new biomarkers which would help to predict the patient progression and prognosis of CRC, thus to give proper recommendations for the clinical therapeutics. 
Table I. Sequencing primers for PCR.

\begin{tabular}{lll}
\hline KRAS & F & TAAGGCCTGCTGAAAATGACT \\
KRAS & R & 5'-Biotin-TTGGATCATATTCGTCCACAA \\
KRAS & S & TTGTGGTAGTTGGAGCT \\
BRAF & F & CATAATGCTTGCTCTGATAGGA \\
BRAF & R & 5'-Biotin-CAATTCTTACCATCCACAAAATG \\
BRAF & S & GGTGATTTTGGTCTAGC \\
PIK3CA-9 & F & AACAGCTCAAAGCAATTTCTACAC \\
PIK3CA-9 & R & 5'-Biotin-GGTATGGTAAAAACATGCTGAGAT \\
PIK3CA-9 & S & AAGCAATTTCTACACGAG \\
PIK3CA-20 & F & GACATTGCATACATTCGAAAGAC \\
PIK3CA-20 & R & 5'-Biotin-GTTTAATTGTGTGGAAGATCCAA \\
PIK3CA-20 & S & AGGCTTTGGAGTATTTCAT
\end{tabular}

F, forward direction; R, reverse direction; $\mathrm{S}$, sequencing.

Table II. Frequencies of KRAS, BRAF and PIK3CA mutations.

\begin{tabular}{|c|c|c|c|c|c|c|}
\hline & \multicolumn{2}{|c|}{ KRAS } & \multicolumn{2}{|c|}{ BRAF } & \multicolumn{2}{|c|}{ PIK3CA } \\
\hline & W & M & W & M & W & M \\
\hline No. & 137 & 63 & 186 & 14 & 175 & 25 \\
\hline Percentage & 68.50 & 31.50 & 93 & 7 & 87.50 & 12.50 \\
\hline
\end{tabular}

$\mathrm{W}$, wild, $\mathrm{M}$, mutation.

\section{Materials and methods}

Study group. Two hundred paraffin-embedded tumor specimens were collected from colorectal cancer patients who underwent resection of primary tumors at Xijing Hospital of Digestive Diseases from 2008 to 2009. The clinicopathological parameters of the 200 cases of the colorectal cancers were collected from surgical and pathological records. Tissue collection and analyses were approved by the Xijing Hospital Public Health Institutional Review Boards.

Genomic DNA extraction, pyrosequencing for KRAS, BRAF and PIK3CA. Genomic DNA was extracted from paraffinembedded tumor specimens using the E.Z.N.A ${ }^{\mathrm{TM}}$ FFPEDNA Kit (Omega Bio-Tech Inc., Doraville, GA, USA). Pyrosequencing assay was used to detect KRAS (exon 1, condon 12 and 13), BRAF (exon 15, V600) and PIK3CA (exon 9, 542 and 545; exon 20, 1044 and 1047) mutations. Sequencing primers for PCR are shown in Table I.

Each PCR mix contained the forward and reverse primers (10 mM, each $0.5 \mu \mathrm{l}), 10 \mathrm{X}$ PCR buffer $5 \mu \mathrm{l}$, dNTP $(2.5 \mathrm{mM})$ $4 \mu \mathrm{l}$, Hot start Taq $0.4 \mu \mathrm{l}, \mathrm{H}_{2} \mathrm{O} 35.6 \mu \mathrm{l}$, and $4 \mu \mathrm{l}$ of template DNA product in a total volume $50 \mu 1$. Mixtures were denatured for $3 \mathrm{~min}$ at $95^{\circ} \mathrm{C}$ and then thermal cycled for $10 \mathrm{sec}$ at $95^{\circ} \mathrm{C}, 20 \mathrm{sec}$ at $56^{\circ} \mathrm{C}$ and $30 \mathrm{sec}$ at $72^{\circ} \mathrm{C}$, repeating the cycle 30 times. A final extension step at $72^{\circ} \mathrm{C}$ for $5 \mathrm{~min}$ completed the program. The PCR products were electrophoresed in an agarose gel to confirm successful amplification. The PCR products were purified and sequenced by Pyrosequencer PyroMark ID system (Biotage AB and Biosystems, Uppsala, Sweden) according to manufacturer's instructions.

We detected KRAS, BRAF and PIK3CA mutations in 200 colorectal cancer samples. The associations of KRAS, PIK3CA and BRAF mutations in colorectal cancer with clinical pathologicoparameters were explored using the $\chi^{2}$ test and Fisher's exact test. P-value $<0.05$ was considered to be statistically significant.

\section{Results}

Frequencies of KRAS, BRAF and PIK3CA mutations in colorectal cancer. We examined KRAS (exon 1, codon 12, 13 and 12.13), BRAF (exon 15, V600E) and PIK3CA (exon 9, 20) mutations in 200 cases of colorectal cancer samples. We detected KRAS mutation in 63/200 patients (31.5\%), BRAF mutation in 14/200 patients (7\%) and PIK3CA mutation in 25/200 (12.5\%) patients. The frequencies of KRAS, BRAF and PIK3CA mutations are shown in Table II, which was similar with previous studies in western countries. Pyrosequencing technology has been shown to be applicable to paraffin-embedded tumor tissue and more sensitive than Sanger dideoxy sequencing. Mutation programs are shown in Figs. 1-3. KRAS mutations were found mostly in codon 12 (GGT-GAT, GGT-GCT, GGT-GTT, GGT-TGT), while codon 13 mutations (GGC-GAC) were fewer than codon 12 mutations. We found a codon 12.13 (GGT-GAT, GGC-GAC) bi-mutation, which has not been reported before (Table III). Table IV shows that the mutation of PIK3CA occurred in exon $9(5428 / 200,5454 / 200)$ and exon 20 (1047 $5 / 200,10498 / 200$ ).

KRAS and PIK3CA mutations, but not BRAF mutation are associated with Dukes' staging. In our study, we classified the patients into 4 stages, as follows: A (T1-2N0M0, 45/200, $22.5 \%)$, B (T3-4N0M0, 93/200, 46.5\%), C (T1-4N1-3M0, 

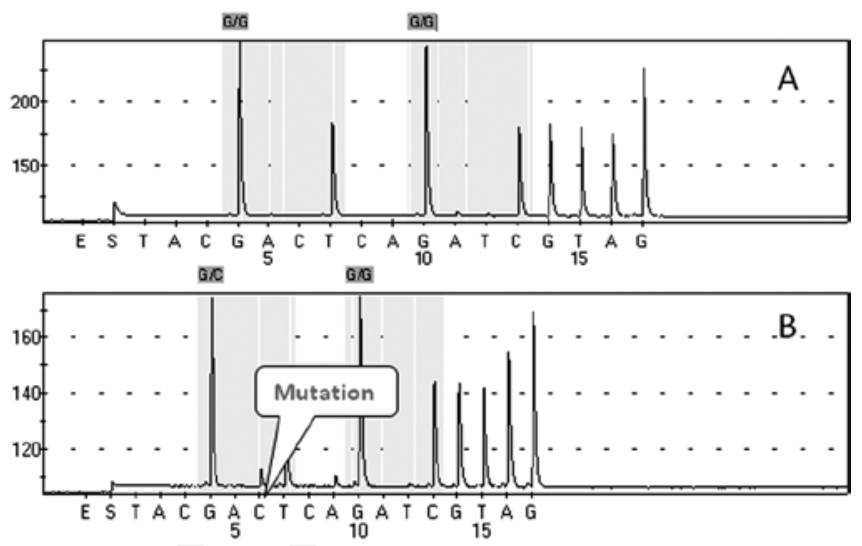

酯 明

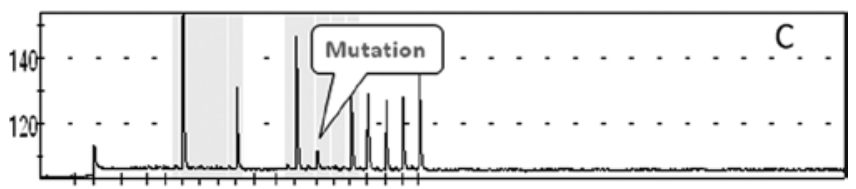

ES T ACGACTCAGATCGTAG

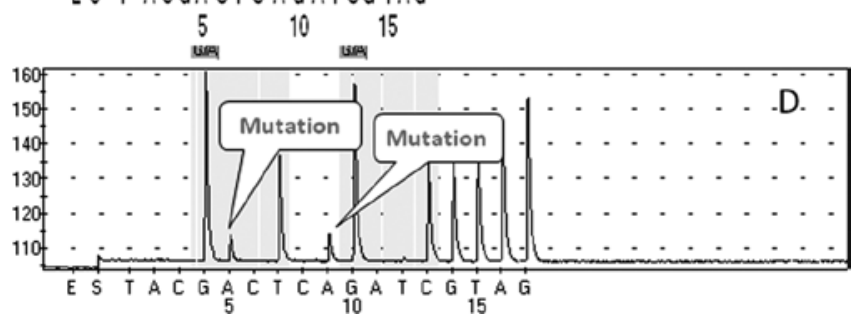

Figure 1. KRAS exon 1 pyrograms. (A) Wild-type exon 1. (B) Codon 12 G-C mutation. (C) Codon 13 G-A mutation. (D) Codon 12 G-A mutation and codon 13 G-A mutation.
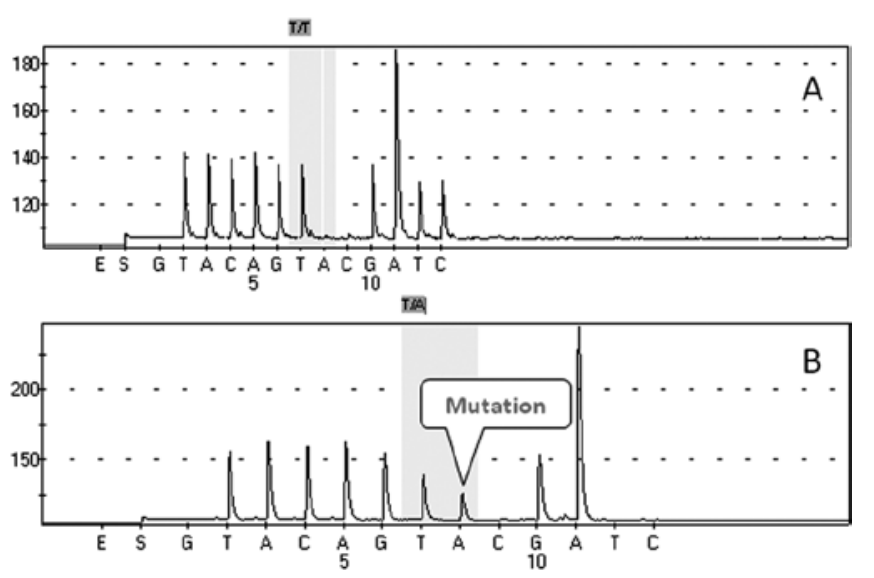

Figure 2. BRAF exon 15 pyrograms. (A) Wild-type exon 15. (B) Codon 600 T-A mutation.

37/200, 18.5\%), D (T1-4N0-3M1, 25/200, 12.5\%). Our study indicated that both KRAS and BRAF mutations are exclusive, but KRAS and PIK3CA mutations are coexistent. We found that KRAS and PIK3CA mutations were significantly associated with Dukes' staging. In contrast, no significant relationship was found between BRAF mutation and Dukes' staging. The higher the colorectal cancer staging, the more KRAS and PIK3CA mutations occurred. The PIK3CA mutations were mostly in $\mathrm{D}$ staging of colorectal cancer patients
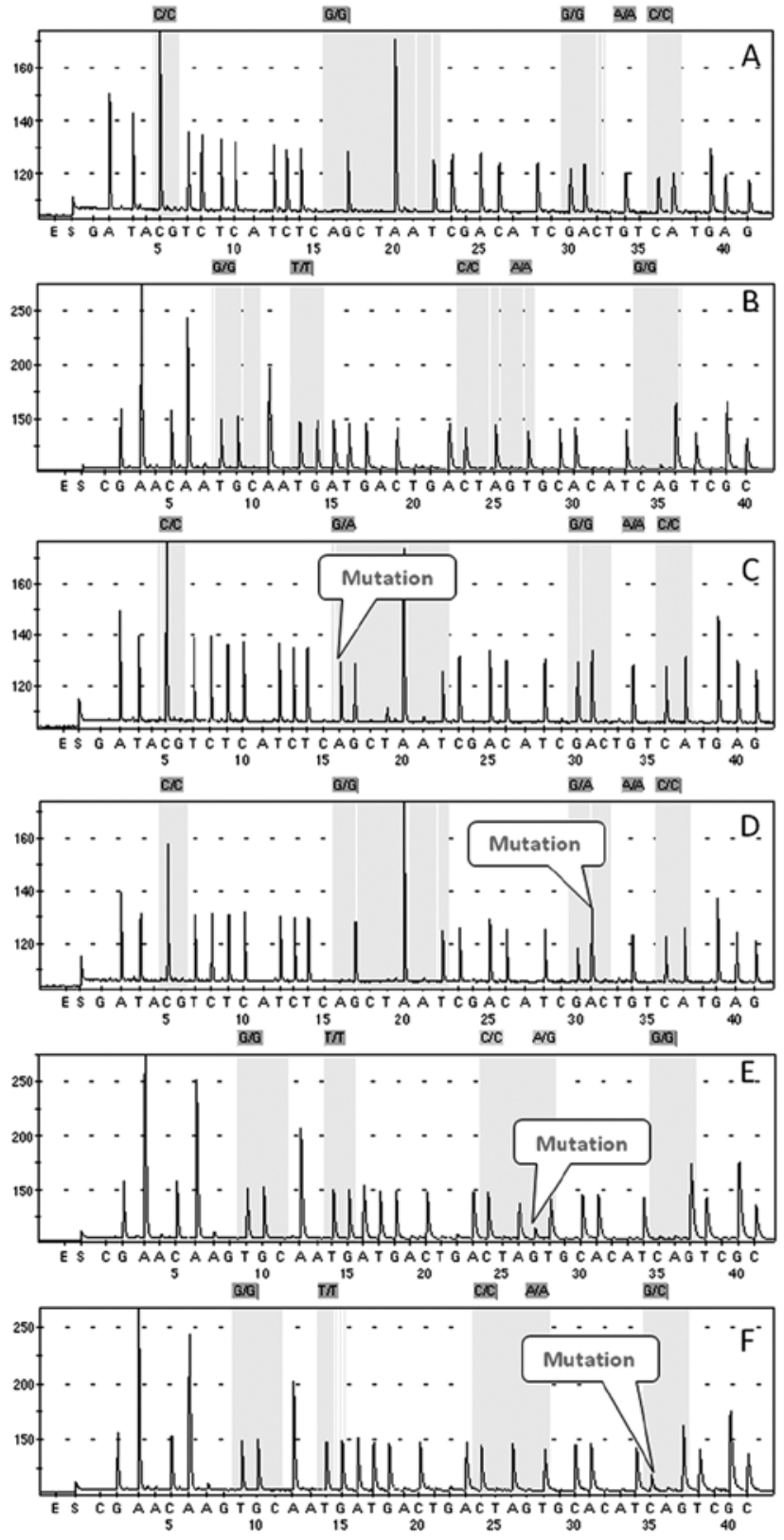

Figure 3. PIK3CA exon 9, 20 pyrograms. (A) Wild-type exon 9. (B) Wildtype exon 20. (C) Exon 9 codon 542 G-A mutation. (D) Exon 9 codon 545 G-A mutation. (E) Exon 20 codon 1047 A-G mutation. (F) Exon 20 codon 1047 A-G mutation.

$(48 \%=12 / 25, \mathrm{P}<0.05)$ (Table V). PIK3CA mutation was more common in KRAS mutated tumors $(22 \%=14 / 63, \mathrm{P}<0.05)$ than KRAS wild-type tumors $(8 \%=11 / 137)$. In addition, our data show no significant relationship between PIK3CA and BRAF mutations.

KRAS and PIK3CA Bi-mutations are significantly elevated in Dukes' D patients. Table VI shows that KRAS and PIK3CA bi-mutations are significantly elevated in Dukes' D patients. When patients were classified into Dukes' D staging, compared with Dukes' A, B and C staging, this showed higher frequencies of KRAS and PIK3CA bi-mutations $(32 \%=8 / 25, \mathrm{P}<0.05)$. These data suggested that colorectal cancers with KRAS and 
Table III. Frequencies of KRAS mutation.

\begin{tabular}{|c|c|c|c|c|c|c|}
\hline & \multicolumn{6}{|c|}{ KRAS mutation } \\
\hline & \multicolumn{4}{|c|}{ Codon 12} & \multirow{2}{*}{$\frac{\text { Codon } 13}{\text { GAC }}$} & \multirow{2}{*}{$\frac{\text { Codon } 12.13}{\text { GAT.GAC }}$} \\
\hline & GAT & GCT & GTT & TGT & & \\
\hline No. & 25 & 5 & 15 & 7 & 10 & 1 \\
\hline Percentage & 12.50 & 2.50 & 7.50 & 3.50 & 5.00 & 0.50 \\
\hline Total (\%) & \multicolumn{4}{|c|}{26.00} & 5.00 & 0.50 \\
\hline
\end{tabular}

W, wild; M, mutation.

Table IV. Frequencies of PIK3CA mutation.

PIK3CA mutation

\author{
Exon 9 \\ Exon 20
}

Codon 539 Codon 542 Codon 545 Codon 546 Codon 1043 Codon 1044 Codon $1047 \quad$ Codon 1049

\begin{tabular}{lllllllll}
\hline No. & 0 & 8 & 4 & 0 & 0 & 0 & 5 & 8 \\
Percentage & 0 & 4.00 & & 2.00 & 0 & 0 & 0 & 2.50 \\
Total (\%) & & & 6.00 & & & & &
\end{tabular}

Codon 542 mutation, GAA-AAA; codon 545 mutation, GAG-CAG; codon 1047 mutation, CAT-CGT(4) CAT-CAG(1); codon 1049 mutation, GGT-GGC.

Table V. Relationship of KRAS, BRAF and PIK3CA mutations with Dukes' staging.

\begin{tabular}{|c|c|c|c|c|c|c|c|c|c|c|c|}
\hline \multirow[b]{2}{*}{ Dukes' } & \multirow[b]{2}{*}{ TNM } & \multirow[b]{2}{*}{$\mathrm{n}$} & \multicolumn{3}{|c|}{ KRAS } & \multicolumn{3}{|c|}{ BRAF } & \multicolumn{3}{|c|}{ PIK3CA } \\
\hline & & & $\mathrm{n}(\%)$ & P-value & $\chi^{2}$ & $\mathrm{n}(\%)$ & P-value & $\chi^{2}$ & $\mathrm{n}(\%)$ & P-value & $\chi^{2}$ \\
\hline A & T1-2N0M0 & 45 & $11(24)$ & 0.002224 & 14.5692 & 0 & 0.1116 & 6 & $2(4)$ & 0.02825 & 9.08 \\
\hline B & T3-4N0M0 & 93 & $28(30)$ & & & $6(6)$ & & & $7(8)$ & & \\
\hline $\mathrm{C}$ & T1-4N1-3M0 & 37 & $8(22)$ & & & $5(14)$ & & & $4(11)$ & & \\
\hline $\mathrm{D}$ & T1-4N0-3M1 & 25 & $16(64)$ & & & $3(12)$ & & & $12(48)$ & & \\
\hline
\end{tabular}

$\chi^{2}$ test for given probabilities.

PIK3CA bi-mutations are more likely to develop into liver metastasis. Besides, our study indicated that KRAS, BRAF and PIK3CA mutations were not significantly correlated with the patient age, gender, tumor location and pathological categories (Table VII).

\section{Discussion}

Colorectal cancer is the third most common human malignant disease in the world. More than $25 \%$ CRC patients present with metastatic disease, with 5-year survival rate less than $10 \%$ (14). Over the past decades, huge progression in molecular biology and cellular biology contributed greatly to the improved understanding of mechanisms of CRC development and progression. It is known that the development of colorectal cancer can be driven by constitutive activation of signaling pathways downstream of the epidermal growth factor receptor (EGFR), such as the RAS-RAF-MAPK and PI3K-PTEN-AKT pathway (5). Such activation can occur via mutations in oncogenes such as KRAS or BRAF of the EGFR-mediated pathway or by PIK3CA mutation or loss of tumor suppressor genes such as PTEN (15). With the development of molecular targeted agents, the therapeutic options for patients with metastatic colorectal cancers (mCRC) have increased. Monoclonal antibodies against EGFR, such as cetuximab or panitumumab, have shown great efficacy in 
Table VI. Relationship of KRAS and PIK3CA bi-mutations with Dukes' staging.

\begin{tabular}{lcccccc}
\hline & & \multicolumn{5}{c}{$\begin{array}{c}\text { KRAS and PIK3CA } \\
\text { bi-mutations }\end{array}$} \\
\cline { 5 - 7 } Dukes' $^{n}$ & TNM & $\mathrm{n}$ & $\mathrm{n}(\%)$ & P-value & $\chi^{2}$ \\
\hline A & T1-2N0M0 & 45 & 0 & & 0.002784 & 14.0909 \\
B & T3-4N0M0 & 93 & 1 & $(1)$ & & \\
C & T1-4N1-3M0 & 37 & 2 & $(5)$ & & \\
D & T1-4N0-3M1 & 25 & $8(32)$ & & \\
\hline
\end{tabular}

$\chi^{2}$ test for given probabilities.

combination with chemotherapy in the first-line treatment of mCRC (16). The EGFR monoclonal antibodies exert their effects by inhibition of binding of the ligand to EGFR and the prevention of subsequent dimerization of the receptor which leads to the activation of several intracellular signal transduction pathways stimulating cell proliferation, neoangiogenesis and inhibiting apoptosis (7). Studies indicate that response to cetuximab is confined to $\mathrm{mCRC}$ patients with wildtype KRAS $(17,18)$. However, there are still some patients with KRAS codon 12 and 13 wild-type tumors who do not respond to anti-EGFR monoclonal antibodies (19). Recently, it was shown that BRAF, PIK3CA mutations are significantly associated with the low response rate (20), but whether these potential predictive biomarkers are validated or not still needs further investigation.

Many studies have reported the KRAS, BRAF and PI3CA mutational status in CRC patients from the West $(5,21)$. However, there is still lack of data from the East. It was reported that colorectal cancers with KRAS mutation are more likely to develop into a polypoid growth (22). However, other studies failed to discover any relationship between gene mutations and clinical characteristics of CRC. Thus, we designed this study aiming to provide data on KRAS, BRAF and PIK3CA mutation status from the East and to explore the relationship between these mutations and clinicopathological parameters, also expecting to find some predictive biomarkers for the risk of colon cancer metastasis.

In our study, 200 cases of colorectal cancer samples were collected and pyrosequencing technology was adopted to explore the mutations of KRAS, BRAF and PI3CA. Pyrosequecing, which has been shown to be more sensitive than regular Sanger sequencing, is a relatively straightforward method for identification of gene mutations in paraffinembedded tumor samples (23). The frequencies of mutations in our samples are compatible with data from previous studies (21,23,24-28). Hence, we deduced that there are no significant differences between the mutation status of KRAS, BRAF and PIK3CA from the West and East.

It was reported that KRAS and PIK3CA mutations have a synergistic effect in activating the PI3K-AKT pathway during colorectal cancer development (28). Previous studies have shown that KRAS and PIK3CA mutation rates in stage I-III and stage IV colorectal cancer and there was an association

Table VII. Relationship of KRAS, BRAF and PIK3CA mutation with clinicopathological parameters.

\begin{tabular}{|c|c|c|c|c|c|c|c|}
\hline Characteristics & $\mathrm{n}$ & $\begin{array}{l}\text { KRAS } \\
\text { mutation } \\
\text { n }(\%)\end{array}$ & P-value & $\begin{array}{c}\text { BRAF } \\
\text { mutation } \\
\mathrm{n}(\%)\end{array}$ & P-value & $\begin{array}{c}\text { PIK3CA } \\
\text { mutation } \\
\mathrm{n}(\%)\end{array}$ & P-value \\
\hline \multicolumn{8}{|l|}{ Gender } \\
\hline Male & 117 & $32(27)$ & \multirow[t]{2}{*}{$>0.05$} & $13(11)$ & \multirow[t]{2}{*}{$>0.05$} & $15(13)$ & \multirow[t]{2}{*}{$>0.05$} \\
\hline Female & 83 & $31(37)$ & & 1 (1) & & $10(12)$ & \\
\hline \multicolumn{8}{|l|}{ Age (years) } \\
\hline$\leq 59$ & 93 & $35(38)$ & \multirow[t]{3}{*}{$>0.05$} & $8(9)$ & \multirow[t]{3}{*}{$>0.05$} & $14(15)$ & \multirow[t]{3}{*}{$>0.05$} \\
\hline $60-69$ & 64 & $19(30)$ & & $3(5)$ & & $5 \quad(8)$ & \\
\hline$\geq 70$ & 43 & $9(21)$ & & $3(7)$ & & $6(14)$ & \\
\hline \multicolumn{8}{|l|}{ Tumor location } \\
\hline Colon (L) & 44 & $15(34)$ & \multirow[t]{3}{*}{$>0.05$} & $7(16)$ & \multirow[t]{3}{*}{$>0.05$} & $5(11)$ & \multirow[t]{3}{*}{$>0.05$} \\
\hline Colon $(\mathrm{R})$ & 48 & $16(33)$ & & $2(4)$ & & $10(21)$ & \\
\hline Rectum & 108 & $32(30)$ & & $5 \quad(5)$ & & $10 \quad(9)$ & \\
\hline \multicolumn{8}{|l|}{ Histological type } \\
\hline Adenocarcinoma & 156 & $44(28)$ & \multirow[t]{5}{*}{$>0.05$} & $13(8)$ & \multirow[t]{5}{*}{$>0.05$} & $18(12)$ & \multirow[t]{5}{*}{$>0.05$} \\
\hline Mucinous adenocarcinoma & 34 & $15(44)$ & & 0 & & $5(15)$ & \\
\hline Signet ring cell carcinoma & 6 & $4(67)$ & & $1(17)$ & & $2(33)$ & \\
\hline Squamous-celled carcinoma & 1 & 0 & & 0 & & 0 & \\
\hline Undifferentiated carcinoma & 3 & 0 & & 0 & & 0 & \\
\hline
\end{tabular}


between PIK3CA and KRAS mutations $(5,29)$. Using 200 colorectal cancer samples, we confirmed that both KRAS and BRAF mutations are exclusive, but KRAS and PIK3CA mutations are coexistent. Our study indicated that KRAS, BRAF and PIK3CA mutations were not significantly correlated with age, gender, tumor location and pathological categories. However, our data revealed significant correlation between KRAS, PIK3CA mutations and Dukes' staging. It was found that KRAS and PIK3CA mutations were significantly associated with Dukes' staging. In contrast, no significant relationship was found between BRAF mutation and Dukes' staging. The higher the colorectal cancer staging, the more frequencies of KRAS or PIK3CA mutations occurred.

Our data showed that PIK3CA mutation is associated with KRAS mutation. PIK3CA mutation was more common in KRAS-mutated tumors than in KRAS wild-type tumors. The PIK3CA mutation mostly occurred in Dukes' D staging of KRAS-mutated tumors. These data suggested that the colorectal cancer with KRAS and PIK3CA bi-mutations are more likely to develop into liver metastasis. However, large-scale studies are needed to confirm the association between KRAS and PIK3CA mutations and the risk of liver metastasis and the patient prognosis.

There were some conflicts as to whether KRAS or PIK3CA is a prognostic factor in colorectal cancer $(30,31)$. In our study, we found strong correlation between KRAS, PIK3CA or bi-mutation and liver metastasis. It is known that patients of mCRC have poor prognosis. Hence, colorectal cancer patients with KRAS, PIK3CA mutation or bi-mutations might have a poor prognosis and KRAS and PIK3CA bi-mutations may become potential predictive and prognostic factors.

Our studies still had some shortages that need to be taken into account when interpreting the results. Firstly, the relatively small sample size might not provide enough statistics to explore the relationship between gene mutations and prognosis. Secondly, the patients' follow-up study is not reported. In further work, we will enlarge our sample size and add the follow-up data from patients who underwent resection and accepted chemotherapy with or without cetuximab. We expect to find whether these gene mutations can accurately predict the response to cetuximab or not, which will provide the necessary adjustment for optimized personalized treatment.

In conclusion, pyrosequencing technology has proven to be a useful method in detecting gene mutations from paraffin-embedded tumor tissues. In 200 colorectal cancer samples, we found that KRAS and PIK3CA mutations were significantly associated with Dukes' staging. In contrast, no significant relationship was found between BRAF mutation and Dukes' staging. The higher the colorectal cancer staging, the more KRAS and PIK3CA mutations occurred. PIK3CA mutations were found mostly in Dukes' D staging of KRASmutated tumors. Colorectal cancer patients with KRAS and PIK3CA bi-mutations are more likely to develop into liver metastasis.

\section{Acknowledgements}

This study was supported by the National Basic Research Program of China (No. 2009CB521705), and the Chinese
National Foundation of Natural Sciences (Nos. 30672399, 30973410 and 30971337).

\section{References}

1. Ikenoue T, Kanai F, Hikiba Y, et al: Functional analysis of PIK3CA gene mutations in human colorectal cancer. Cancer Res 65: 4562-4567, 2005.

2. Cengel KA, Voong KR, Chandrasekaran S, et al: Oncogenic K-Ras signals through epidermal growth factor receptor and wild-type $\mathrm{H}$-Ras to promote radiation survival in pancreatic and colorectal carcinoma cells. Neoplasia 9: 341-348, 2007.

3. Tsareva SA, Moriggl R, Corvinus FM, et al: Signal transducer and activator of transcripton 3 activation promotes invasive growth of colon carcinomas through matrix metalloproteinase induction. Neoplasia 9: 279-291, 2007.

4. Chiang CT, Way TD, Tsai SJ and Lin JK: Diosgenin, a naturally occurring steroid, suppresses fatty acid synthase expression in HER 2 overexpressing breast cancer cells through modulating Akt, mTOR and JNK phosphorylation. FEBS Lett 581: 5735-5742, 2007.

5. Barault L, Veyries N, Jooste V, et al: Mutations in the RAS-MAPK, $\mathrm{PI}(3) \mathrm{K}$ (phosphatidylinositol-3-OH kinase) signaling network correlate with poor survival in a population-based series of colon cancers. Int J Cancer 122: 2255-2259, 2008.

6. Yarden Y and Sliwkowski MX: Untangling the ErbB signalling network. Nat Rev Mol Cell Biol 2: 127-137, 2001.

7. Scaltriti $\mathrm{M}$ and Baselga J: The epidermal growth factor receptor pathway: a model for targeted therapy. Clin Cancer Res 12: 5268-5272, 2006.

8. Siena S, Sartore-Bianchi A, Di Nicolantonio F, Balfour J and Bardelli A: Biomarkers predicting clinical outcome of epidermal growth factor receptor-targeted therapy in metastatic colorectal cancer. J Natl Cancer Inst 101: 1308-1324, 2009.

9. Kiaris $\mathrm{H}$ and Spandidos DA: Mutations of ras genes in human tumours. Int J Oncol 7: 413-421, 1995.

10. Benvenuti S, Sartore-Bianchi A, Di Nicolantonio F, et al: Oncogenic activation of the RAS/RAF signaling pathway impairs the response of metastatic colorectal cancers to antiepidermal growth factor receptor antibody therapies. Cancer Res 67: 2643-2648, 2007.

11. Lievre A, Bachet JB, Le Corre D, et al: KRAS mutation status is predictive of response to cetuximab therapy in colorectal cancer. Cancer Res 66: 3992-3995, 2006.

12. Frattini M, Signoroni S, Pilotti S, et al: Phosphatase protein homologue to tensin expression and phosphatidylinositol-3 phosphate kinase mutations in colorectal cancer. Cancer Res 65: 1122-1127, 2005.

13. Freeman DJ, Juan T, Reiner M, et al: Association of K-ras mutational status and clinical outcomes in patients with metastatic colorectal cancer receiving Panitumumab alone. Clin Colorectal Cancer 7: 184-190, 2008.

14. Jemal A, Clegg LX, Ward E, et al: Annual report to the nation on the status of cancer, 1975-2001, with a special feature regarding survival. Cancer 101: 3-27, 2004.

15. Sartore-Bianchi A, Martini M, Molinari F, et al: PIK3CA mutations in colorectal cancer are associated with clinical resistance to EGFRtargetd monoclonal antibodies. Cancer Res 69: 1851-1857, 2009.

16. Van Cutsem E, Kohne CH, Hitre E, et al: Cetuximab and chemotherapy as initial treatment for metastatic colorectal cancer. $\mathrm{N}$ Engl Med 360: 1408-1417, 2009.

17. Tol J, Dijkstra JR, Klomp M, et al: Markers for EGFR pathway activation as predictor of outcome in metastatic colorectal cancer patients treated with or without cetuximab. Eur J Cancer 46: 1997-2009, 2010.

18. Qiu LX, Mao C, Zhang J, et al: Predictive and prognostic value of KRAS mutations in metastatic colorectal cancer patients treated with cetuximab: a meta-analysis of 22 studies. Eur J Cancer 46: 2781-2787, 2010.

19. Allegra CJ, Jessup JM, Somerfield MR, et al: American Society of Clinical Oncology provisional clinical opinion: testing for KRAS gene mutations in patients with metastatic colorectal carcinoma to predict response to anti-epidermal growth factor receptor monoclonal antibody therapy. J Clin Oncol 27: 2091-2096, 2009.

20. De Roock W, Claes B, Bernasconi D, et al: Effects of KRAS, BRAF, NRAS, and PIK3CA mutations on the efficacy of Cetuximab plus chemotherapy in chemotherapy-refractory metastatic colorectal cancer: a retrospective consortium analysis. Lancet Oncol 11: 753-762, 2010. 
21. Samuels Y, Wang Z, Bardelli A, et al: High frequency of mutations of the PIK3CA gene in human cancers. Science 304: 554-561, 2004.

22. Chiang JM: Role of K-ras mutations in colorectal carcinoma. Cancer Lett 126: 179-185, 1998.

23. Miyaki M, Iijima T, Yamaguchi T, et al: Mutations of the PIK3CA gene in hereditary colorectal cancers. Int J Cancer 121: $1627-1630,2007$.

24. Glarakis IS, Savva S and Spandidos DA: Activation of the ras genes in malignant and premalignant colorectal tumors. Oncol Rep 5: 1451-1454, 1998.

25. Ogino S, Kawasaki T,Brahmandam M, et al: Sensitive sequencing method for KRAS mutation detection by Pyrosequencing. J Mol Diagn 7: 413-421, 2005.

26. Velho S, Oliveira C, Ferreira A, et al: The prevalence of PIK3CA mutations in gastric and colon cancer. Eur J Cancer 41: 1649-1654, 2005.
27. Kato S, Iida S, Higuchi T, et al: PIK3CA mutation is predictive of poor survival in patients with colorectal cancer. Int J Cancer 121: 1771-1778, 2007.

28. Benvenuti S, Frattini M, Arena S, et al: PIK3CA cancer mutations display gender and tissue specificity patterns. Hum Mutat 29: 284-288, 2008

29. Tol J, Nagtegaal ID and Punt CJ: BRAF mutation in metastatic colorectal cancer. N Engl J Med 361: 98-99, 2009.

30. Richman SD, Seymour MT, Chambers P, et al: KRAS and BRAF mutations in advanced colorectal cancer are associated with poor prognosis but do not preclude benefit from oxaliplatin or irinotecan: results from the MRC FOCUS trial. J Clin Oncol 27: 5931-5937, 2009

31. Ogino S, Nosho K, Kirkner GJ, et al: PIK3CA mutation is associated with poor prognosis among patients with curatively resected colon cancer. J Clin Oncol 27: 1477-1484, 2009. 